This document is published in:

Cabestani, J. et al. (eds.) (2011) Advances in Computational Intelligence: 11th International Work-Conference on Artificial Neural Networks, IWANN 2011, Torremolinos-Málaga, Spain, June 8-10, 2011, Proceedings, Part II.

(Lecture Notes in Computer Science, 6692). Springer, 653-660. DOI: http://dx.doi.org/10.1007/978-3-642-21498-1_82

(C) 2011 Springer-Verlag Berlin Heidelberg 


\title{
Context-Awareness at the Service of Sensor Fusion Systems: Inverting the Usual Scheme
}

\author{
Enrique Martí, Jesús García, and Jose Manuel Molina \\ Applied Artificial Intelligence Group, Universidad Carlos III de Madrid, \\ Av. de la Universidad Carlos III, 22, 28270, Colmenarejo, Madrid, Spain \\ emarti@inf.uc3m.es, jesus.garcia@inf.uc3m.es, molina@ia.uc3m.es, \\ http://www.giaa.inf.uc3m.es
}

\begin{abstract}
Many works on context-aware systems make use of location, navigation or tracking services offered by an underlying sensor fusion module, as part of the relevant contextual information. The obtained knowledge is typically consumed only by the high level layers of the system, in spite that context itself represents a valuable source of information from which every part of the implemented system could take benefit. This paper closes the loop, analyzing how can context knowledge be applied to improve the accuracy, robustness and adaptability of sensor fusion processes. The whole theoretical analysis will be related with the indoor/outdoor navigation system implemented for a wheeled robotic platform. Some preliminary results are presented, where the context information provided by a map is integrated in the sensor fusion system.
\end{abstract}

Keywords: sensor fusion, navigation, indoor, outdoor, context-aware, particle filter, software agent.

\section{Introduction}

Sensor Fusion is a process that consists in combining observations provided by several sensors about an entity of interest, so that the information finally obtained is better - in some sense - than what could be inferred by taking each of the sensors alone. This task is just a part of the more general concept of Data Fusion which, among other particularities, is not limited to sensing information. Location and tracking of dynamic objects [11][13][8][2][5] can be accounted as one of the most important applications of sensor fusion. Solving this problem requires a clear specification of, at least, what has to be estimated (variables of interest about the observed system), data provided by sensors, and how both system state and sensor readings are related. Fusion performance can be benefited of any additional information, such as a mathematical model of observed system dynamics. Fusion processes in real scenarios are, however, affected by a variety of external factors that cannot be accounted while modeling the problem, either because are subject to uncontrolled changes over time, or because they are even unknown to us. 
To overcome that hurdle, fusion systems should be able to detect relevant changes in their environment, and adapt themselves for achieving the best performance: this is, context-aware sensor fusion. Most of the existing literature about context-aware applications featuring location and/or navigation follow this scheme in the opposite direction: fusion module performs location, and the obtained result is employed as a position-based context for higher-level applications [1][12].

The discipline of Data Fusion represents an example of fusion processes responsive to their environment. In the JDL model [9] for data fusion systems, the 4 level (Process Refinement) describes how to use the acquired information to feedback the lower levels by means of, for instance, sensor retasking or model modification. The goal of this paper is to analyze the direct application of contextual information to sensor fusion tasks. Many of the provided examples are based on the platform used in the experimental part, which was introduced in [10] and is briefly described in section 2. It consists in an autonomous robot that performs indoor and outdoor navigation using a variety of onboard and external sensors, enabling the scenario for representing a full scale fusion problem. Following that, section 3 conduct a theoretical analysis regarding the applicability of contextual information in sensor fusion processes. It begins covering the topic of modeling environment for context acquisition and processing. Immediately afterwards, a second part identifies the parts in a sensor fusion process where the obtained contextual information can be applied. Finally, some preliminary results using the described platform are presented in section 4 . They show how sensor fusion can benefit from the use of contextual information either improving accuracy or reducing the computational burden of selected algorithms. Some remarks and conclusiones are given in the last section.

\section{Sample Scenario}

The scenario selected as reference for the analysis conducted in next section is the problem of combined indoor/outdoor navigation: estimation of position, orientation and dynamics of a robot, which is equipped with onboard sensors but also features communication capabilities with other entities that will serve as external sensors. From the architectural point of view, this navigation system is organized in layers in order to maximize its flexibility. Each tier plays a different role in the process of acquiring and transforming information into something useful for the final data sink: a Particle Filter which fuses all the information into the most likely estimation. One of the strongest reasons for selecting such a solution is to provide a reasonably complete scenario that does not limit the performed theoretical analysis.

Figure 1 contains a schematic view of the system. Information flows top-down in the diagram, with sensor data represented by small triangles. Upper levels are in charge of capturing information either by means of sensors physically attached to the platform or by exchanging data with external intelligent entities. The sensor abstraction layer is in charge of managing physical sensors as well 
as providing a unified vision of external information sources. The applications of contextual information to sensor fusion described in section 3 involve acting over this layer.

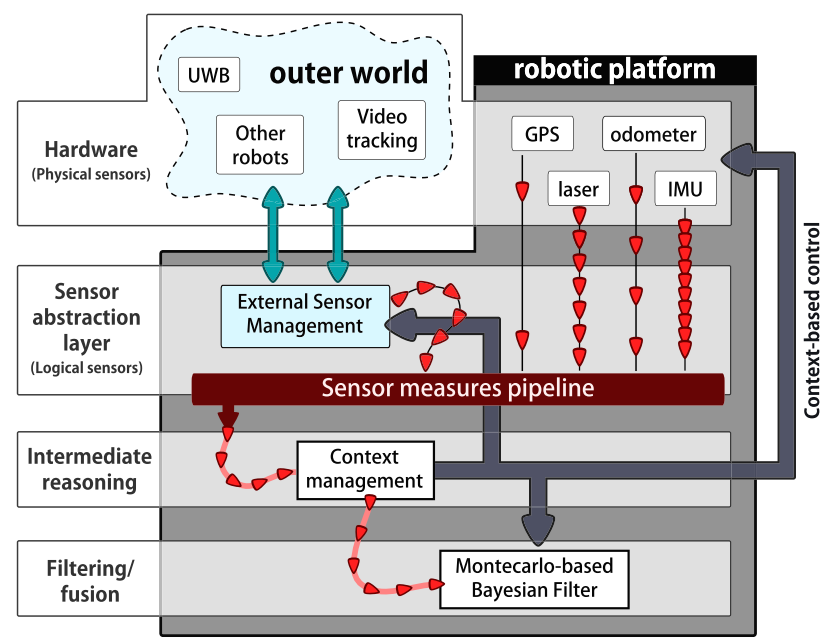

Fig. 1. Architecture of the proposed navigation system for an individual mobile platform

The Intermediate reasoning layer receives and process raw sensor measures. The operations hosted by this level range from adapting sensing information to meet the various requirements of the filter, to more advanced inferences as deriving context information from the available readings. The diagram shows only the box corresponding to context reasoning for the sake of clarity. The last level contains simply a filtering-capable algorithm for integrating the incoming data.

\section{Theoretical Analysis}

This section is divided in two parts. The first one reviews different representations of environment for extracting contextual information, and the second details where and how this information can be used within a sensor fusion system.

\subsection{Knowledge about Environment and Context Representation}

Let us define the environment of an application as the multidimensional space where it operates, including other variables which an influence on the problem being solved. Those variables can be categorized in different ways, for instance, if we focus on what that knowledge is referred to, we can discern between information about the environment itself and information about the different entities populating it. Inside the first category we can account for several types of information attending to its nature. 
If we are talking about either continuous or discrete valued variables that have a defined value for every point of the environment (i.e. fields), then the most straightforward representation is a map. Examples of this type of variables are ambient temperature, obstacle location or signal/noise level for a certain electromagnetic emission.

Some information which is not likely to be mapped, can be represented by statements instead (i.e. declarative knowledge). The weather is a perfect example of propositional context knowledge: sunny or rainy condition, current wind speed, etc. are factors to take into account in sensor fusion becasue they can affect the performance of some devices. Knowledge about external entities also lays in the field of statement-based information, but is a bit trickier because it tends to involve complex reasoning processes. Entity-related knowledge can be classified in two general families: feature and relational knowledge.

Among the many examples of feature knowledge we can cite identity, position, activity and utility. Although the extraction of such features can involve complex data structures and intrincated processing schemes - some of them still an open research field, as activity recognition-, they can be represented as value tuples or simple labels once have been determined. Relational knowledge describes the different interactions or links between entities. Ontologies for entity and graphs for groupal activity recognition can be enclosed in this category.

\subsection{Applying Context to Sensor Fusion}

The acquired contextual information can be injected in differents places of a sensor fusion system. The two principal insertion points are the set of sensors and the fusion algorithm. The first category, acting over the sensors, includes at least four uses of context information: sensor selection, modification of capture parameters, modification (correction) of raw sensing data, and finally sensing data augmentation (complete it with new information). The two first types are commonly known as sensor retasking in terminology of distributed multisensor data fusion. Nonetheless, they can be important features also in centralized, simple sensor fusion systems. For instance, selection plays an important role when dealing with redundant sets of sensors.

Sometimes direct observations can provide the required context, as in the case of fusing video and infrared sensors: in spite that both provide spatial information about non-occluded surfaces in the environment, poor lighting conditions discards a video camera as an effective sensor, while an infrared sensor can be affected by colors and reflection angles. Both effects can be detected using the sole video input. Another example is shown in figure 2. The motion of the rover in a rough floor cause vibrations which spoil the measures, as seen around second 9. On the other hand, some effects are not as easy to detect. A sustained magnetic interference can bias the readings of a magnetometer, but figuring out the existence of this problem requires further estimations processes and context data. 


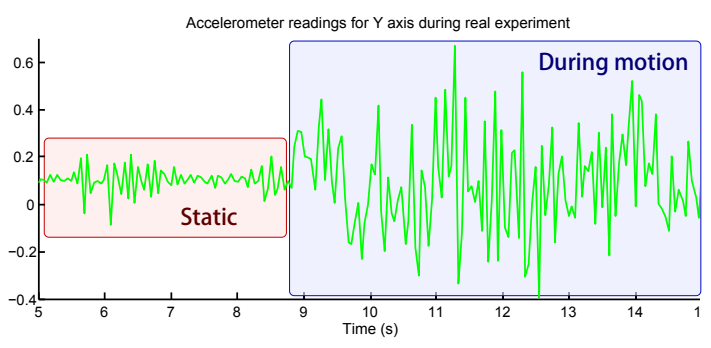

Fig. 2. Vibrations due to robot motion have a harsh effect on inertial unit measures

Regarding the modification of capturing parameters, we can cite changing the orientation of directional sensors such as PTZ video cameras. Existing algorithms for visual attention [7] and tracking can provide the information needed for the change of parameters.

The third option is to modify the sensed data to correct undesired effects. As an example, mobile entities can degrade the performance of map matching algorithms, because they affect laser readings. However, the context can be used to identify and remove the spurious beam hits. Finally, sensor data can be augmented by including additional data to be considered in the fusion process, as the confidence in a measure or a label indicating how to process it.

The second entry point for context information in sensor fusion systems is the proper fusion algorithm, by means of selection - change algorithm - and modification - manipulation of parameters - . Examples of algorithm selection can be employing a Particle Filter for indoor navigation, where walls and other obstacles make the problem highly nonlinear, but switching to a simpler and less costly approach such as least squares or a Kalman-like filter in open spaces. Multiple model systems and particle filters with adaptive population size constitute examples of algorithm modification.

\section{Experiments on Navigation}

This part of the paper gathers empirical results obtained with the platform described in section 2 . A first subsection will thoroughly describe the configuration employed in the experiments, immediately followed by the obtained results along with an analysis on them.

\subsection{Navigation System Setup}

The experiments presented in this paper have been reproduced in laboratory from both simulated and real data. The real data has been obtained in controlled experiments where the robot was been equipped with a GPS sensor with meter-level precission and an inertial measurement unit (IMU). With simulation purposes, GPS measures are assumed to suffer a random gaussian-like noise with standard deviation of 1 meter. 
The baseline navigation algorithm relies in a Particle Filter which performs loosely coupled fusion of the two proposed sensors. This approach will be compared with a similar system that also includes the information of a map. It must be noted that the available set of sensors do not allow the implementation of map matching techniques [6][3] that, provided with an almost perfect map, can result in an outstanding positioning performance. Instead, the map is used to discarding particles that move into a wall, as done in [4].

The system is tested in a very simple porch-like scenario. It presents obstacles to be mapped, while being an almost open space with avaialble GPS signal. The robot navigates in a relatively reduced space that, provided the low accuracy of GPS measures, makes the problem more difficult to solve.

\subsection{Obtained Results}

The conducted experiments compare navigation performance with and without a map of obstacles. Using standard sensing conditions, with update rates around $30 \mathrm{~Hz}$ for the IMU and $1 \mathrm{~Hz}$ for the GPS, the map-less navigation algorithm usually results in an average positioning error of 0.4 meters. This represents a $65 \%$ error decrease compared with the average $1.2 \mathrm{~m}$ error of bare GPS measures.

Figure 3 shows the filtered trajectory for one of the runs. The slash-marked path represents the true trajectory of the robot, while the track of dense small circles represent the Particle Filter estimation. GPS measures are marked as large circles, while the cloud of points with attached lines represent the position and orientation of particles at the end of the simulation. When using a map, the algorithm not only worsens its position estimation ( 0.6 meters, $50 \%$ improvement), but also spoils the continuity of the estimated trajectory with sudden jumps, as shown in figure 4 . They are a direct effect of particle anihilation together with biased GPS measures. The real improvement, as in many sensor fusion systems, comes by the hand of degraded sensor performance. For the following experiments, degraded sensing conditions were assumed: IMU readings feature a noise characteristic of rough terrain, and GPS measures lower the update rate to 0.2

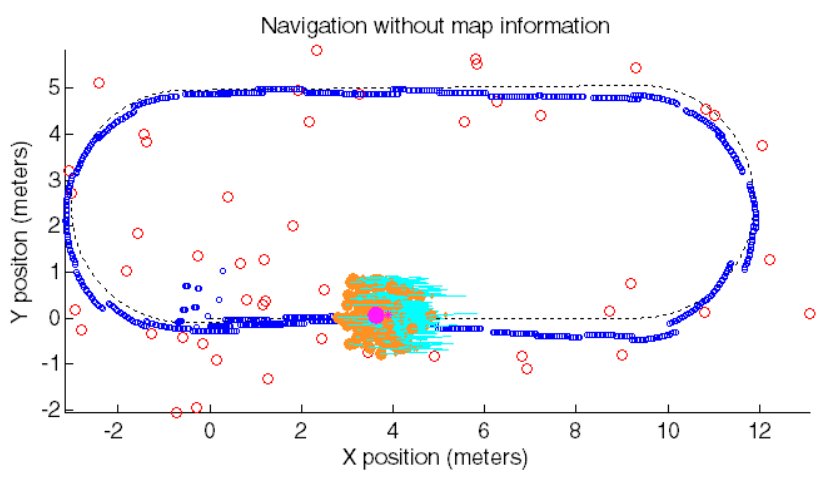

Fig. 3. Navigation without map, assuming best sensing conditions 
$\mathrm{Hz}$ as in the case of occlusions. Under these conditions, taking into account map information leads to slightly better results. The baseline navigation algorithm have a mean position error of 1.2 meters, same as GPS measures, while including the map results in about 0.9 meters, although its stimate is still less smoother than the basic version.

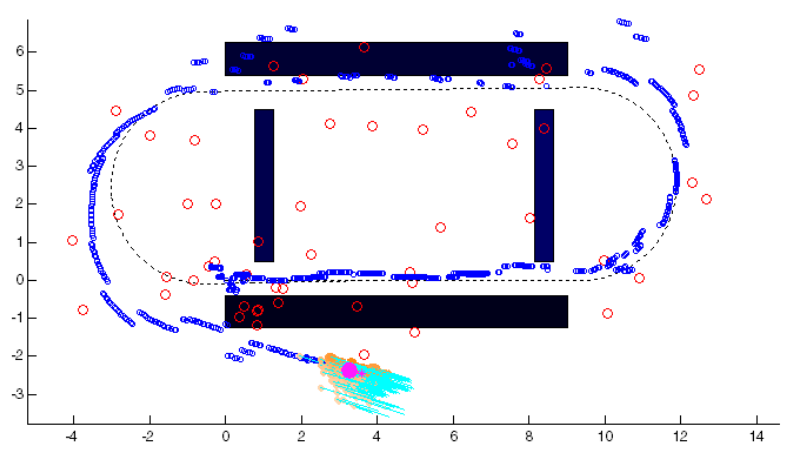

Fig. 4. Navigation with map, assuming best sensing conditions

In spite that the performed experiments are quite reduced, they show a fundamental fact: context information, when adecquately integrated into sensor fusion systems, can improve their robustness under conditions of degraded sensing performance. This is of vital importance for systems pretending to be autonomous and work unattended for long periods of time.

\section{Conclusions}

The contribution of this paper is two-fold. On the one hand, the theoretical analysis in section 3 tries to conciliate the worlds of Data Fusion and contextaware applications. It can be seen that all provided examples, which have been related to the sample scenario but can also be found in existing literature, solve problems using sensor fusion systems and context information. The problem is that authors are usually very focused in the context part and do not make use of those formalisms developed within Data Fusion field. Integrating both disciplines can lead not only to better results, but also to a faster progress thanks to not reinventing existing concepts. The other contribution is an scheme defining how artificial intelligence applications can feedback their sensor fusion modules in order to improve their results. Some preliminary experiments on indoor/outdoor navigation are also presented, where the simple use of a map provides the necessary context to improve location accuracy under degraded sensor performance. The obtained results are far from being spectacular in aboslute terms, but it is important to take into account that the goal was to test if even a very weak use of context information could serve to improve the performance of a sensor fusion system. 


\section{Acknowledgements}

This work was supported in part by Projects ATLANTIDA, CICYT TIN200806742-C02-02/TSI, CICYT TEC2008-06732-C02-02/TEC, SINPROB, CAM MADRINET S-0505/TIC/0255 DPS2008-07029-C02-02.

\section{References}

1. Bernardos, A.M., Tarrio, P., Casar, J.R.: A data fusion framework for contextaware mobile services. IEEE, Los Alamitos (2008)

2. Castanedo, F., García, J., Patricio, M.A., Molina, J.M.: Data fusion to improve trajectory tracking in a Cooperative Surveillance Multi-Agent Architecture. Information Fusion 11(3), 243-255 (2010)

3. Dellaert, F., Fox, D., Burgard, W., Thrun, S.: Monte Carlo localization for mobile robots. In: Proceedings 1999 IEEE International Conference on Robotics and Automation (Cat. No.99CH36288C), pp. 1322-1328 (February 2001)

4. Evennou, F., Marx, F., Novakov, E.: Map-aided indoor mobile positioning system using particle filter. In:IEEE Wireless 2005 (2005)

5. Gómez-Romero, J., Patricio, M.A., García, J., Molina, J.M.: Ontology-based context representation and reasoning for object tracking and scene interpretation in video. Expert Systems with Applications 38(6), 7494-7510 (2010)

6. Gustafsson, F., Gunnarsson, F., Bergman, N., Forssell, U., Jansson, J., Karlsson, R., Nordlund, P.-J.: Particle filters for positioning, navigation, and tracking. IEEE Transactions on Signal Processing 50(2), 425-437 (2002)

7. Horaud, R., Knossow, D., Michaelis, M.: Camera cooperation for achieving visual attention. Machine Vision and Applications 16(6), 1-2 (2005)

8. Jing, L., Vadakkepat, P.: Interacting MCMC particle filter for tracking maneuvering target. Digital Signal Processing 20(2), 561-574 (2010)

9. Liggins, M.E., Llinas, J., Hall, D.L.: Handbook of Multisensor Data Fusion: Theory and Practice, 2nd edn. CRC Press, Boca Raton (2008)

10. Martí, E., García, J., Molina, J.: Opportunistic multisensor fusion for robust navigation in smart environments. In: Proceedings of CONTEXTS 2011 Workshop User-Centric Technologies and Applications, Springer, Heidelberg (2011)

11. Nemra, A., Aouf, N.: Robust INS/GPS Sensor Fusion for UAV Localization Using SDRE Nonlinear Filtering. IEEE Sensors Journal 10(4), 789-798 (2010)

12. Subercaze, J., Maret, P., Dang, N.M., Sasaki, K.: Context-aware applications using personal sensors. In: Proceedings of the ICST 2nd International Conference on Body Area Networks, p. 19 (June 2007)

13. Wendel, J., Trommer, G.: Tightly coupled GPS/INS integration for missile applications. Aerospace Science and Technology 8(7), 627-634 (2004) 